statement made by these authors, was found to contain a large concentration of hydroxylamine. This solution, like hydroxylamine and unlike oxime, on addition of sodium nitroprusside and a little caustic soda, turns red on heating. Like hydroxylamine it oxidises hæmoglobin to methæmoglobin. It precipitates cuprous oxide from an alkaline solution of copper sulphate, and the titration of this solution with Fehling solution reveals the presence of about $87 \mathrm{mgm}$. of free hydroxylamine. We find therefore that about 66 per cent oxime was decomposed. liberating a corresponding amount of hydroxylamine.

On testing this solution with nickel it was found to contain only 29 per cent of undecomposed dimethylglyoxime, which conforms fairly well with the previous estimation of the free hydroxylamine.

These results show that $0 \cdot 018 M$ solution of oxime treated by the method of Sevag and Maiweg gives approximately $0.024 M$ solution of hydroxylamine. In other words, the molar concentration of hydroxylamine (a well-known catalase inhibitor) in Sevag and Maiweg's solution, at the end of their manipulation, was higher than the initial concentration of the oxime. It is not surprising, therefore, that their solution had such a powerful inhibitory action on catalase.

D. Krilla .

Molteno Institute,

E. F. Hartree.

University, Cambridge.

${ }^{1}$ Sevag, M. G., and Maiweg, Lore, Naturwissenschaften, 22, 561; 1934.

\section{Passage of Very Fast Protons through Matter}

IT is now generally assumed in nuclear theories ${ }^{1}$ that the interaction between a neutron and a proton is of the form

$$
-J(r) S
$$

where $J(r)$ is some function of the distance $r$ between them, and $S$ is the operator which interchanges the space co-ordinates but not the spin of the two particles. Such an interaction has some important consequences in the passage of very fast protons through matter.

We consider a collision in which the neutron is initially at rest, and the proton moving with a large momentum such that the wave-length is small compared to the effective radius of the interaction $J(r)$. Then, as Wick ${ }^{2}$ has pointed out, with an exchange interaction of the type (1), most of the neutrons are thrown in the forward direction. (With a nonexchange interaction, most of the protons would be scattered in the forward direction.) To calculate the magnitude of the collision cross section we must make some assumption about the form of $J(r)$, of which we know very little. Assuming $J(r)$ to be of the form

$$
J(r)=a e^{-b r}
$$

the effective cross section, calculated by the Born approximation, for the scattering of the neutron in the solid angle $d \omega$ making an angle $\theta$ with the original direction of the proton is

$$
\begin{aligned}
& \frac{256 \pi^{4}}{h^{4}} \frac{a^{2} b^{2}}{\left\{b^{2}+\frac{4 \pi^{2}}{h^{2}}\left|\underline{p_{1}}-\underline{p_{0}}\right|^{2}\right\}^{4}} \times \\
& \frac{p_{1}{ }^{3} E_{0} E_{1}}{p_{0}\left\{p_{1}+\frac{E_{1}}{E_{0}+M c^{2}-E_{1}}\left(p_{1}-p_{0} \cos \theta\right)\right\}^{2} c^{4}} d \omega
\end{aligned}
$$

where $p_{0}=M v / \sqrt{ }\left(1-v^{2} / c^{2}\right)$ is the initial momentum of the proton, $v$ its velocity, $M$ the mass of the proton or neutron assumed equal, and $E_{0}=c \sqrt{ }\left(p_{0}{ }^{2}+M^{2} c^{2}\right)$ the initial energy of the proton. $E_{1}, p_{1}$ are the energy and momentum of the neutron given by the conservation of energy and momentum for a particle scattered at an angle $\theta$. The effective cross section for the scattering of the neutron in any angle less than $\theta$ is

$$
q(\theta)=\frac{64 \pi^{3}}{3} \frac{E_{0}^{2}}{c^{2} p_{0}^{2}} \cdot \frac{a^{2} b^{2}}{h^{2} c^{2}}\left[\frac{1}{b^{6}}-\frac{1}{\left\{b^{2}+\left(\frac{2 \pi p_{0}}{h}\right)^{2} \sin ^{2} \theta\right\}^{3}}\right]
$$

where we have neglected terms of the order $(h b / 2 \pi M c)^{2}$. When $2 \pi p_{0} / h \gg b$, (3) has a very strong maximum in the forward direction, and in this case the mean angle $\bar{\theta}$ at which the neutrons are ejected is

$$
\bar{\theta}=\frac{1}{\sqrt{ } 2} \cdot \frac{h b}{2 \pi p_{0}},
$$

and the neutron has on the average an energy less than the original energy of the proton by an amount $\delta$, where

$$
\delta=\frac{1}{16 \pi^{2}} \cdot \frac{h^{2} b^{2}}{M} \text {. }
$$

Taking $a=1.4 \times 10^{-4} \mathrm{erg}, b=6.8 \times 10^{12} \mathrm{~cm} .^{-1}$, being the values determined by Wick $^{3}$ from the stability of oxygen, the total cross section for an encounter $q$, got by neglecting the last term in square brackets in (4), is $0.16 \times 10^{-24} \mathrm{~cm}^{2}, \bar{\theta}=1 \cdot 1^{\circ}$, and $\delta \approx 0.47 \times 10^{6}$ e.v., for a proton with $E_{0}=5 \times 10^{9}$ e.v. $\approx 5.4 M c^{2}$, such as might possibly occur in cosmic radiation.

Thus, such a proton in its passage through matter will go on until it collides with a neutron and gives nearly all its energy to the neutron. The neutron will travel until it collides with a proton in the material as given by (4), (5), (6), and the proton which is thus ejected will have very nearly the same energy as the original proton, and travel in very nearly the same direction. Further, for protons of such high energies we should expect to be able to treat the neutrons in nuclei as free, so that, using the cross section given above, the mean 'range' of a proton in lead is about $1.5 \mathrm{~cm}$. , and that of the neutron about $2 \cdot 3 \mathrm{~cm}$., as the lead nucleus contains 82 protons and about 125 neutrons. Therefore in going through a metre of lead there will be roughly twentyfive changes from proton to neutron and back, and the total energy loss due to ionisation of the emerging proton will be about half that to be expected theoretically since more than half the distance will be travelled by neutrons.

We may remark, that with the values of $a$ and $b$ assumed above, an accurate calculation ${ }^{3}$ of the cross section for the collision of neutrons of energy roughly $3.8 \times 10^{6} \mathrm{e} . \mathrm{v}$. with protons gives a result which is nearly four times larger than the cross section found by Chadwick. Our results may therefore be too large by a factor four.

The above expressions are further inaccurate inasmuch as $(a)$ there is no relativistic wave equation for the proton, and $(b)$ the interaction energy ( 1 ) can be represented by a potential $J(r)$ probably only in non-relativistic approximation. One might hope that the correct relativistic treatment would reduce the total cross section given above.

Gonville and Caius College, H. J. Внавна. Cambridge. Nov. 10.

${ }^{2}$ w. Heisenberg, Z. Phys., 80, 587; 1933. E. Majorana, $Z$. Phys., 82, 137 ; 1933.

G. C. Wick, Z. Phys., 84, 799; 1933.

${ }^{3}$ G. C. Wick, Nuovo Cimento, 11, 235 ; 1934. 